\title{
Predictive Feed Forward Stereo Processing
}

Stephen B. Pollard, John Porrill and John E. W. Mayhew

\author{
AI Vision Research Unit \\ University of Sheffield \\ Sheffield S10 2TN \\ England
}

\begin{abstract}
This paper describes the preliminary stages in the development of a predictive feed-forward (PFF) stereo based tracking module. The object of the module is to exploit the spatio-temporal coherence that exists in a sequence of stereo images in the context of providing a visual control mechanism for a mobile vehicle with uncertainty in position. PFF provides a method by which the representation of a $3 D$ scene can be maintained and evolved over time. Furthermore, quickening strategies can utilise the spatio-temporal coherence by exploiting previously obtained depth values and approximate trajectory information in order to accelerate the process that actually achieves the stereo correspondences.
\end{abstract}

Much research in computer vision has been developed in snapshot mode, concentrating attention on a single image or a small number of frames obtained either in synchrony or from a short movie sequence, the computational overheads involved in the analysis of a long sequence of images having proved prohibitive. It has been assumed, albeit implicitly, that algorithms developed in this way would, given appropriate parallel computer architectures, eventually be able to perform in real time on continuous image sequences. That is, the whole process involved in the recovery of scene descriptions would begin afresh upon each image frame and that some [not fully defined] extra module would be responsible for maintaining an evolving model of the environment based upon these descriptions.

In the context of the control of a mobile robotic vehicle it is important to distinguish between the twin goals of obtaining an accurate model of the environment and determining the current position within it. Hence dynamic vision can be decomposed into [at least] two important modules:

1) The maintenance of an accurate and, as far as possible, topologically complete scene model. This will include: the combination of multiple views $[1,2]$ to give more complete and robust data; the identification of and inclusion of novel (not previously seen) features; the determination of free space within which the robot can move.

2) The use of visual tracking to provide the control signal required to navigate an autonomous vehicle through an unstructured/partially structured environment using as beacons a subset of the scene features (perhaps identified outside the tracking module itself).

The visual through-put and temporal response required by each task is very different. For example, when using visual feed back as a control mechanism it will be necessary to provide a much higher sample rate than that at which the model of the environment needs to be updated. Furthermore, the actual elapsed time

This research was supported by SERC project grant no. GR/E 49616 awarded under the ACME programme in collaboration with IBM UK, Scientific Centre, Winchester. at which control information is made available will be more crucial than the time taken to build and update the environmental model. For these reasons it would seem natural to build a dynamic vision system for autonomous navigation with two loosely connected components with very different temporal rates that reflect their required behaviours. This approach follows roughly the ideas of a subsumption architecture [3].

Here we shall consider the dynamic stereo tracking problem associated with following a path through a scene based upon a subset of features chosen to act as beacons. The adopted strategy is a simple one (see figure 1). After a start up phase which involves full stereo processing of the scene, the recovery of 3 dimensional geometrical primitives and some topological/statistical completion, a small number of beacons are chosen. The processing of subsequent frames is performed in the context of the existing description of the beacons (taking into account any available trajectory information) via a cycle of back-projection feature tracking and geometrical consistency to give an updated estimate of their 3 dimensional location (and conversely the estimate of the robot's position with respect to them).

In current implementations of the system, scene descriptions are limited to straight line geometrical primitives and hence the application domain is limited to well carpentered worlds. It is of course our intention to extend the primitive base to allow a wider area of application. We are fortunate in this regard in that the stereo matching algorithm we employ [4] works at the level of the edgel (rather than polygonal approximation) and is reasonably generic in this regard.

Figure 1

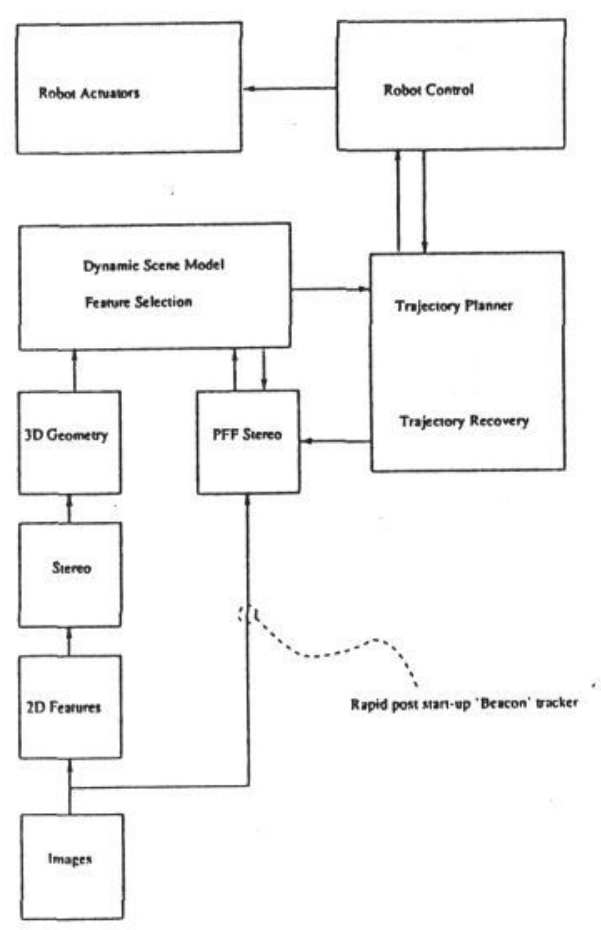




\section{PATH FOLLOWING}

Consider the abstract design of a vehicle control system (VCS) which is able to steer along the path $P$ based upon the availability of positional information (for example the PID controller discussed in the experimental section below). Assume also that the controller is able to provide its own estimate of position (either direct odometry from motor feedback or upon the basis of an internal model) in the absence of an external source. This system can be represented as the following function

$$
T_{t+1}=\operatorname{VCS}\left(T_{b}, P, t, t+1\right)
$$

That is the expected location at time $t+1$ is determined from the location $T_{t}$ and the path $P$. Similarly the position at a future time $t^{\prime}$ is given by

$$
T_{t^{\prime}}=\operatorname{VCS}\left(T_{t}, P, t, t^{\prime}\right)
$$

Suppose we have an updated estimate of the of our position at time $t^{\prime}$ from visual feedback, call it $T^{*}$, then the most obvious form of visual feedback is to substitute $T^{*}{ }^{*}$ for $T_{t}$ in the vehicle controller. However it is necessary to make allowance for the time delay required to process the visual data. If say the visual feedback for image frames captured at time $t^{\prime}$ is not available until $t^{\prime \prime}$ then the best estimate for position at time $t^{\prime \prime}$, and the one substituted into the controller, will in fact be

$$
T_{t^{\prime \prime}}=T_{t}^{*}+\left(T_{t^{\prime \prime}}-T_{t^{\prime}}\right)
$$

that is the the best estimate of the current location is given by the last known position for which a visual estimate exists plus a component since that time based upon vehicle control.

Current research is aimed at developing more sophisticated strategies for estimating position, employing forms of the Kalman filter to optimally combine estimates from the delayed visual data with current vehicle control information.

\section{PFF: PREDICTIVE FEED-FORWARD STEREO}

One can imagine a number of quickening strategies to utilise the spatio-temporal coherence that exists in a sequence of stereo images in order to accelerate the process that actually achieves the stereo correspondences. The most obvious approach is to assume that the local change in disparity that occurs between two closely spaced frames in a stereo sequence will be of limited magnitude. This would allow the search for stereo correspondence to be restricted to a small disparity window determined from the range of disparities found in the immediate neighbourhood of the frame considered previously.

More sophisticated control strategies can also be considered. Estimates of the current trajectory of the robot and/or the objects in the [segmented] scene can be exploited in order to give a first estimate (one which assumes partial rigidity) of the locations of features that are expected to arise in subsequent views. Such hypotheses could be confirmed/modified through a process of back projection. Given sufficient subtlety the process of scene prediction would allow novel features to be identified rapidly and attended to. Furthermore a small number of focus features, once their initial predictions have been verified, could be used to give more accurate estimates of the best partial scene transformations and further reduce the cost of matching (particularly when trajectory estimates are poor or not given).

Figure 2 is a block diagram representing the PFF stereo mechanism used here. The cycle is as follows:

(i) Predict location of line features to be used as beacons (given by their last known position plus an estimate of tra- jectory) and, through a process of back projection, their left and right hand image descriptions.

(ii) Identify the most likely candidate for each line in the left and right image using the line tracker module.

(iii) Recombine tracked left and right image features to give updated geometrical description of line in 3D.

(iv) Assume rigid transformation of scene (robot only moving) and find optimal transformation taking predicted geometry into updated geometry.

(v) Update actual geometrical description of beacons and refine estimate of location with respect to them.

It is possible that at stage (ii) one or other image primitive resulting from the back projection is either not tracked or tracked incorrectly resulting in missing or incorrect 3D data at stage (iii). However as an estimate of the transformation is available at stage (v) it is possible to update the geometry of all beacons, including the spurious ones, and hence little fallout of data is experienced by the process. This provides an advantage over strategies that allow independent $2 \mathrm{D} / 3 \mathrm{D}$ tracking of individual 2D/3D primitives.

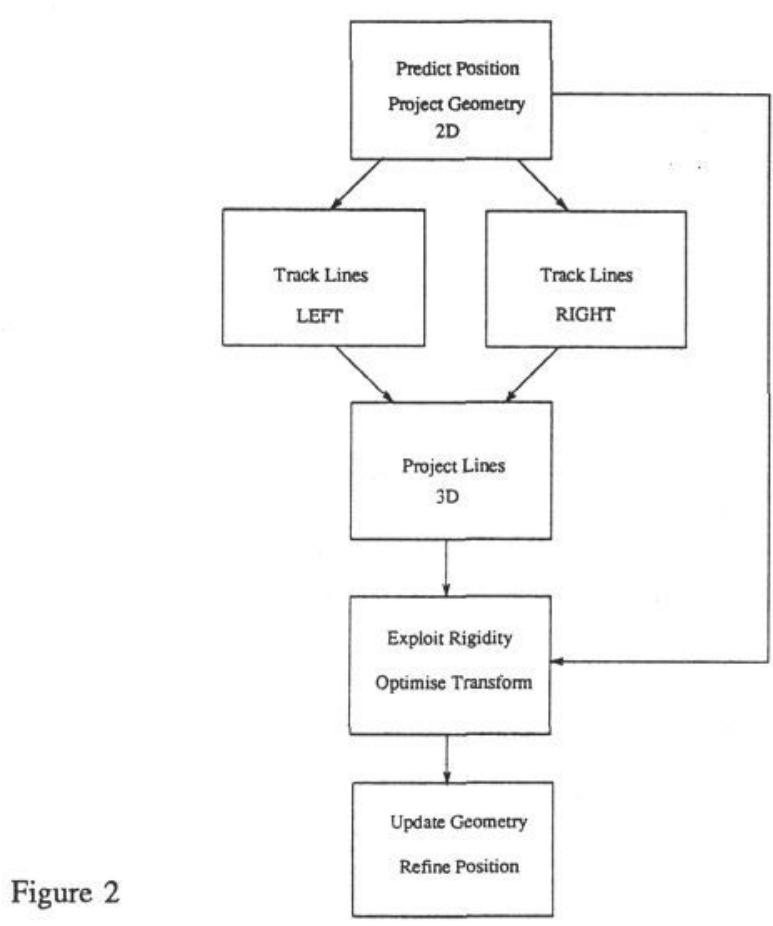

\section{D Feature Verification/Tracking}

The consistent line closest to a predicted line can be obtained using a rapid verification cycle. Consistency is defined in terms of orientation and, if required, the contrast of the line. Contrast being viewpoint specific can only be exploited as a consistency measure if the predicted viewpoint is similar to the viewpoint at which the contrast of the line was last measured and provided a catastrophe of viewpoint has not been encountered. Furthermore it is important that the contrast of a line is specified in a viewpoint independent fashion.

The 2D tracking procedure is currently performed as follows:

1) $\mathrm{N}$ equally spaced points along the predicted line are chosen.

2) For each such point the closest consistent candidate edge point in direction orthogonal to the predicted line is located. 
3) The set of edge points is searched heuristically for the maximum set that are consistent with a single straight line that is compatible with the orientation constraint.

4) Orthogonal regression is used to fit a straight line to the selected edge points (if more than three such points exist).

5) The chosen line is extended along its length by incremental prediction and verification.

The $\mathrm{N}$ points along each line are chosen to be at least a pixel apart along the line. If $\mathrm{N}$ is made constant for all lines (over a threshold length) it is possible to search for each line in a time independent of its length.

Candidate edge points are located using an algorithm that is essentially an incremental one dimensional version of the Canny edge detector [5]. First the image intensity orthogonal to the edge is arranged as a one dimensional image array. These image intensities could be located in the true orthogonal direction employing forms of intensity interpolation. However, we find it sufficient simply to quantise the orthogonal direction as either horizontal, vertical or one of the diagonals. As a result we may be searching for an edge in a direction that is up to 22.5 degrees from the true orthogonal direction and hence may expect the measure of contrast of the edge to be affected accordingly.

(a)

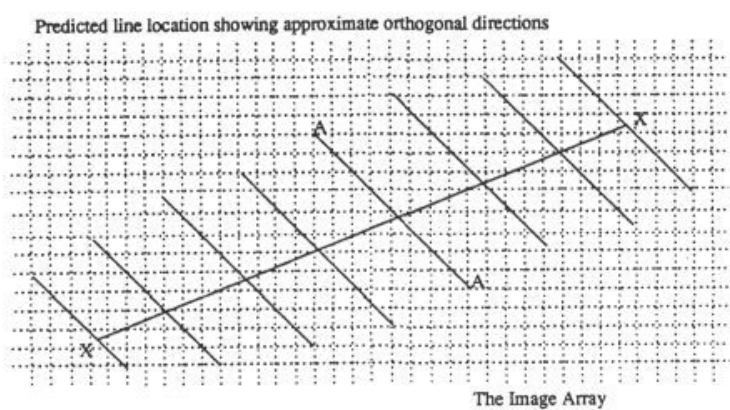

(b)

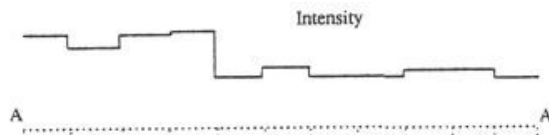

GAUSS

(c)

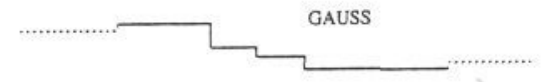

(d)

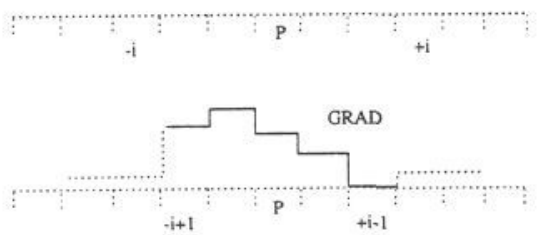

Figure 3.

The search for the closest candidate edge begins at its predicted location (call it P). Then the following algorithm is performed to find the closest consistent edge point (see also figure 3 ):

i) In an iterative fashion pixels to either side of the predicted location that lie at incrementally increasingly disparate locations are considered (in the order $\mathrm{P}-\mathrm{i}$ then $\mathrm{P}+\mathrm{i}$; where $\mathrm{i}$ is the current increment).

ii) Gaussian profile convolution is performed over the (1D) neighbourhood of the pixel and stored as GAUSS[P-i] and GAUSS[P+i] for pixels $\mathrm{P}-\mathrm{i}$ and $\mathrm{P}+\mathrm{i}$.

iii) Gradient values are computed for pixels $\mathrm{P}-\mathrm{i}+1$ and $\mathrm{P}+\mathrm{i}-1$ according to the differences GAUSS[P-i+2]-GAUSS[P-i] and GAUSS[P+i]-GAUSS[P+i-2] respectively and stored as GRAD[P-i+1] and GRAD[P+i-1].

iv) Processing continues until either a limit is reached and no edge is returned or the first suitable maxima in the gradient value is located. The latter is the case if a gradient value has the appropriate sign and approximate magnitude and the condition

|GRAD[max-1]|<|GRAD[max]|

$|\mathrm{GRAD}[\max +1]|<=\mid \mathrm{GRAD}[\max ]$

and

holds (where max is either $\mathrm{P}-\mathrm{i}+2$ or $\mathrm{P}+\mathrm{i}-2$ ).

v) If an appropriate maximum is found, its location is given to a sub-pixel value by approximating it to the maximum in the quadratic fit through the gradient values of this pixel and its immediate neighbours.

The search for the set of points that is consistent with the best straight line has the following form (illustrated in figure 4). Approximations to the line are considered in turn by choosing ordered triplets of candidate edge points. The line is approximated by the difference in the first and third edge locations. Only if the mid point of the triple is compatible with the line description (within a predefined small threshold) and the line itself is consistent with any orientation constraint, is the hypothesis considered further. Each approximation is ranked on the basis of the number of other candidate edge points that could lie upon it (within the threshold). The first hypothesis that is found to be above acceptance threshold (or the best if non are above threshold) forms the basis of the best consistent set. In the event that no hypothesis includes more than 3 edge points then an exhaustive search commences. Each pair of edge points are considered in turn until one provides a suitably good basis for acceptance.

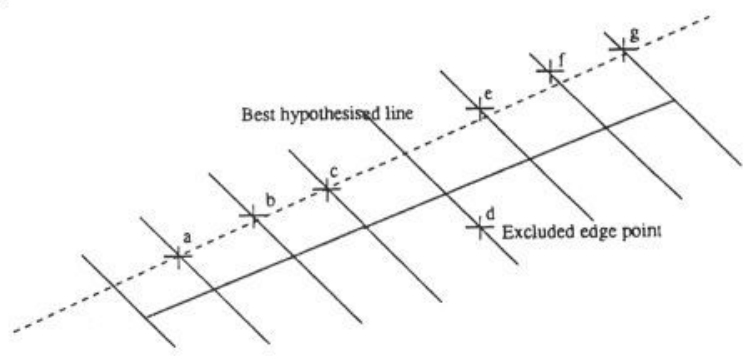

(a)

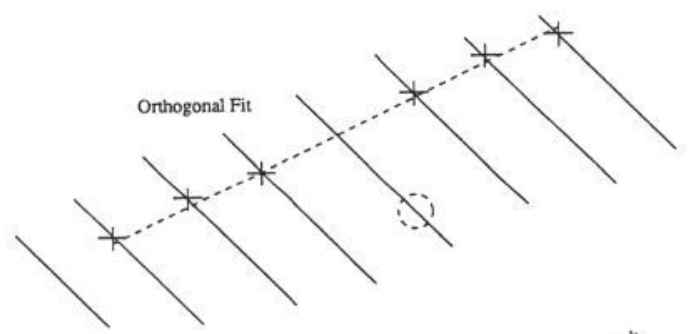

(b)

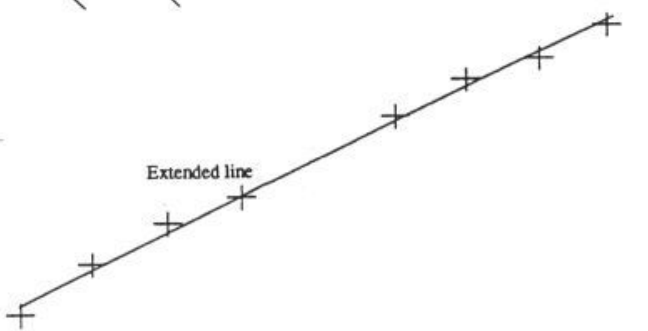

(c)

Figure 4. In (a), the three edge points $a, b$ and $c$ form the basis of the best candidate approximation to a line. Edge points $e, f$ and $g$ are also consistent with the extension of the line ac. Figure (b) shows the results of orthogonal regression to these points. In (c) the final extension of this line is shown. 
It would be possible of course to include within a similar searching strategy the possibility of ambiguity at each edge location. The current approach of finding only the candidate edge location closest to the predicted location could be replaced, at the expense of computational complexity, with one that recovers all suitable edge hypothesis in the neighbourhood. In such a scheme the selection of actual edge locations consistent with a single line interpretation (again at increased computational complexity) would be postponed. However we have found the former strategy sufficient for our purposes.

Once a consistent set of edge points has been identified it is repeatedly fit using orthogonal regression $[6,7]$ and extended to include new edge points that are compatible with it. Extension is first performed over the set of candidate edges not already included in the best consistent set. Further extension is possible beyond the extremities of the current line approximation by recourse to controlled use of the local edge location algorithm discussed above.

\section{Exploiting Rigidity}

Given a set of hypothetical matches between predicted beacon descriptions (in 3D) and their tracked counterparts it is possible to exploit the rigidity assumption in order to obtain a correction for the current estimate of the position of the stereo system, and also determine those pairings which are correct. Rigidity is an assumption that is often used in model matching to determine a consistent set of matches between features of a known object model and features recovered from the scene. The assumption is valid here only if the objects that compose the scene are stationary or that all the beacons arise from a single rigid object. It is possible, however, to imagine developments of such a system that will allow the scene to be segmented at run time on the basis of consistent clusters in transformation space.

The strategy we have adopted is based upon [8] and is as follows. Suppose we have obtained an initial estimate of the correct transformation then it is possible to examine the set of potential matches (predicted to hypothesised) to identify just those that are consistent with it. Only if, after transformation, two lines could occupy the same region of space within the error bounds are they considered to be consistent. The best new transformation, in the least squares sense, consistent with the set of candidate line pairings can now be computed. The process of estimation and consistency checking continues until the set of consistent matches is not added to (note that consistent matches are never deleted from this set to prevent oscillatory behaviour). If this transformation is considered good enough, that is it includes a minimum of 4 matches or half the number of candidate matches if that is greater, it is accepted.

The first estimate to the transformation is obtained from the whole set of potential matchings. If this approach does not result in an acceptable transformation it is necessary to search the set of potential transformations until a suitable candidate transformation results. Fortunately, because of the small set of beacons that are used, coupled to the fact that they are restricted to a single match and the tracking process is robust, the combinatorics of this search do not prove too costly. Each pair of possible matches is allowed to hypothesise a potential transformation. These are investigated in turn using matches for the longest primitives first (these should be most robustly matched).

\section{Optimal Position Updating}

The result of the stereo location of a straight edge $\lambda_{0}$ (ignoring endpoint information) is the position $\mathbf{p}_{0}$ of a point on the line and its unit direction vector $\mathbf{v}_{0}$. If we extend $v_{0}$ to a basis by choosing $b \nu 1, \mathbf{v}_{2}$, then any nearby line $\lambda$ can be described by position $\mathbf{p}$ and direction $\mathbf{v}$ where

$$
\begin{aligned}
& \mathbf{p}=\mathbf{p}_{0}+p_{1} \mathbf{v}_{1}+p_{2} \mathbf{v}_{2} \\
& \mathbf{v}=\mathbf{v}_{0}+v_{1} \mathbf{v}_{1}+v_{2} \mathbf{v}_{2}
\end{aligned}
$$

The map

$$
\lambda \rightarrow \mathbf{x}=\left(p_{1}, p_{2}, v_{1}, v_{2}\right)^{t}
$$

is then a $C^{\infty}$ local coordinate chart on the line manifold near $\lambda_{0}$. To describe the errors of measurement we give the probability distribution of the perturbation 4-vector $\mathbf{x}$ between the measured line $\lambda_{0}$ and its true position $\lambda$. On the assumption of approximate normality this is adequately described by the expected error

$$
E[\mathbf{x}]=\overline{\mathbf{x}}
$$

and the $4 \times 4$ measurement covariance matrix $S$

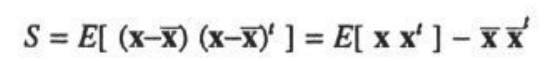

We assume that our stereo measurement process is unbiased so that $\overline{\mathbf{x}}=0$. Methods for estimating the covariance $S$ are outlined in $[9,2]$.

Suppose the camera frame changes between two stereo views by an estimated rotation $R_{0}$ and translation $\mathrm{t}_{0}$. Coordinates $\mathrm{p}$ in the old frame are related to $\mathbf{p}^{\prime}$ in the new by

$$
\mathrm{p}=R_{0} \mathrm{p}^{\prime}+\mathrm{t}_{0}
$$

Rigid transformations $(R, \mathrm{t})$ close to $\left(R_{0}, \mathrm{t}_{0}\right)$ can be approximated by composing this with a second small rigid motion with angular vector $\boldsymbol{\omega}$ and translation vector $\boldsymbol{\tau}$

$$
p \rightarrow p+\omega \times p+\tau
$$

Errors in the transformation between two frames can be described by the covariance of the correction 6-vector $(\omega, \tau)$.

Suppose a line has estimated location and error covariance $\left(\mathrm{p}_{0}, \mathbf{v}_{0}, S\right)$ in the old frame and is matched with $\left(\mathbf{p}_{0}^{\prime}, \mathbf{v}_{0}^{\prime}, S^{\prime}\right)$ in the new frame, the estimated rotation and translation and error covariance between frames being $\left(R_{0}, \mathrm{t}_{0}, S^{\prime \prime}\right)$. The required correction vectors described above for the two lines and the motion can be amalgamated into a $14=4+4+6$ dimensional state vector $\mathbf{X}$ with covariance matrix

$$
S_{0}=\left(\begin{array}{ccc}
S & 0 & 0 \\
0 & S^{\prime} & 0 \\
0 & 0 & S^{\prime \prime}
\end{array}\right)
$$

The condition that the corrected lines are the same in the true frame is

$$
\begin{aligned}
& \mathbf{v}-\mathbf{v}^{\prime \prime}+\omega \times \mathbf{v}^{\prime \prime}+\tau=0 \\
& \left(p-\mathbf{p}^{\prime \prime}+\omega \times \mathbf{p}^{\prime \prime}+\tau\right)-\left(\mathbf{p}-\mathbf{p}^{\prime \prime}+\omega \times \mathbf{p}^{\prime \prime}+\tau\right) \cdot \mathbf{v} \mathbf{v}=0
\end{aligned}
$$

If the four independent components of these two equations are projected out by taking scalar products with $\mathbf{v}_{1}$ and $\mathbf{v}_{2}$ and the results are linearised in the small corrections, we obtain four scalar constraints on the fourteen components of $\mathbf{X}$ of the form

$$
z+\mathbf{h}^{t} \mathbf{X}=0
$$

Our initial estimate of $\mathbf{X}$ is $\mathbf{X}_{0}=0$ with covariance $S_{0}$. The optimal correction to impose a constraint as above is 


$$
\begin{aligned}
& S_{1}=S_{0}-\frac{\left(S_{0} \mathbf{h}\right)\left(S_{0} \mathbf{h}\right)^{t}}{\mathbf{h}^{t} S_{0} \mathbf{h}} \\
& \mathbf{k}=\frac{S_{0} \mathbf{h}}{\mathbf{h}^{t} S_{0} \mathbf{h}} \\
& \mathbf{X}_{1}=\mathbf{X}_{0}-\mathbf{k}\left(z+\mathbf{h}^{t} \mathbf{X}_{0}\right)
\end{aligned}
$$

(note that the correction is described by an 'innovation' term proportional to the error of the old estimate, this is typical of a Kalman filter). The increase in residual is

$$
\varepsilon_{1}=\varepsilon_{0}+\frac{\left(z+\mathbf{h}^{t} \mathbf{X}_{0}\right)^{2}}{\mathbf{h}^{t} S_{0} \mathbf{h}}
$$

The four constraints can be applied sequentially to obtain simultaneously the optimal corrections to the position in the old frame, the position in the new, and the transformation between frames.

If we want to test the plausibility of a match given the a priori information the maximum likelihood test treats the total increase in residual $\varepsilon$ as a $\chi^{2}$ on four degrees of freedom.

The new covariance matrix gives estimates of the error in the three corrections in blocks down the diagonal, and the correlations between corrections are stored off the diagonal. In some applications all this information will be retained, but here we either want to improve our motion estimate or our estimate of the old line position, and we can drop the additional components of the state vector and its covariance.

This constraint application procedure forms the basic unit of a two pass procedure for improving the motion estimate and updating the beacon model. The initial motion estimate is assumed to have near infinite covariance. The state vectors for the first two beacons are appended and the constraints imposed. This is repeated for the whole list of matched beacons, only the motion estimate and its covariance being updated.

A second pass is made down the list with the new motion estimate, updating only the position of the beacons in the old frame. This two pass procedure is sub-optimal, but the optimal procedure involves amalgamating all the beacon corrections into a single long state vector and is computationally unattractive for this application.

Figure 5 .

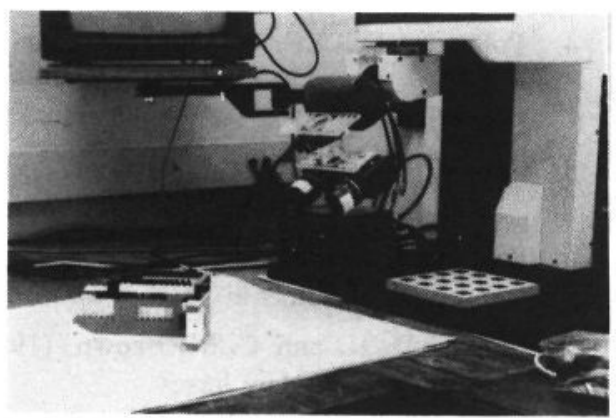

\section{EXPERIMENTAL RESULTS}

Currently we do not have access to a mobile vehicle suitable to test our algorithms or the computing power to implement them in real time. As an alternative we have developed a simple test bed for experimentation which utilises a robot arm to provide an eye in hand stereo rig to simulate a very slow moving vehicle. Figure 5 shows the experimental set up. An UMI robot (of educational rather than industrial specifications) holds a miniature virtual vehicle consisting of a pair of Panasonic WV-CD50 CCD cameras mounted on a fixed stereo rig. The cameras are positioned with optical centers approximately $9 \mathrm{~cm}$ apart and with a slightly asymmetric convergent gaze of approximately 15 degrees fixating a workspace some $35 \mathrm{~cm}$ distant. The light-weight Sony TV lenses have effective focal lengths of approximately $17.5 \mathrm{~mm}$ and subtend a visual angle of about 30 degrees. Recovery of the approximate relationship between the coordinate system used by the robot hand and that used by the stereo system is achieved by making a number of known movements of gripper and noting the resultant transformation of the stereo rig with respect to a fixed calibration tile.

\section{Vehicle Simulation and Control}

The low level robot control system employed to simulate the vehicle is designed to embody some of the features that would present problems for the control of a real vehicle. The motion achieved by the simulated vehicle is quasi continuous with a path constrained to be roughly parallel to the ground plane. The vehicle has state parameters of heading, curvature and speed. Only curvature and speed can be set externally; heading is the result of previous state and is initialised straight ahead. In this way the vehicle roughly simulates a steerable device. The vehicle simulator runs at $5 \mathrm{~Hz}$ (the limiting response time of the robot).

The vehicle control system is of PID design with an update frequency of $2 \mathrm{~Hz}$. Consider the 2D plane which includes the path $P$, within it the position vector $x$ describes the location of the vehicle, $\theta$ the heading angle and $v$ the velocity. Assuming that $x$ does not lie on, or $\theta$ along, the path then it is necessary to update the state parameters to set a course to rejoin the path some distance ahead. A PID controller with critical damping and bandwidth $\lambda$ (characteristic length $L=v / \lambda$; the distance to rejoin the path) is given by

$$
\text { curvature }=-\frac{3}{L} q-\frac{3}{L^{2}} p-\frac{1}{L^{3}} \int p d x
$$

where $p$ is the perpendicular distance from $x$ to $P$ and $q$ is the difference between heading angle and the tangent to the path.

a
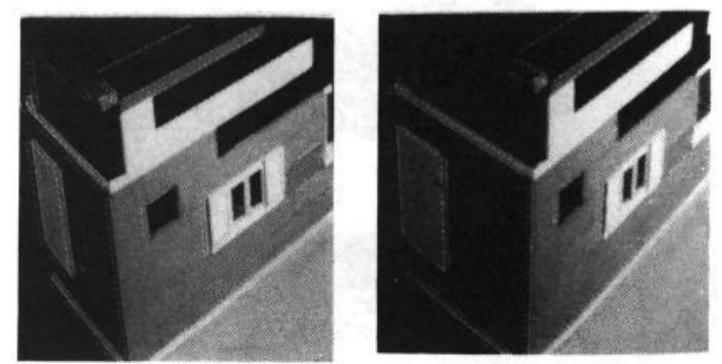

Figure 6
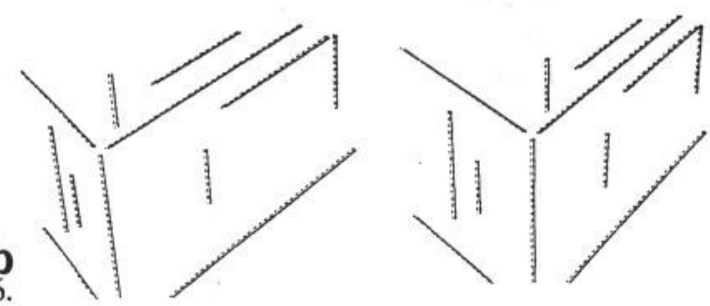

\section{Performance Examples}

The ability of PFF stereo to overcome positional error is illustrated in Figure 6. In part (a) line features for the previously acquired 3D geometry are displayed (dashed) over a new image. Updated line features that result from the correction of position achieved by the PFF process are shown as solid lines. In (b) a subset of 3D line features selected as beacons for rapid visual servo are shown. For clarity, they are not shown over the origi- 
nal image. Running on a Sun $3 / 60$, the total elapsed time take to process a frame and track the beacons was less than 10 seconds (excluding the time to acquire the images) compared to over 90 seconds for full frame stereo.

Figure 7 illustrates the path following scenario. In (a) the path is shown in the $3 \mathrm{~d}$ world coordinates of the vision system (the reconstructed scene description is also shown rendered for completed surfaces). The path is approximately $15 \mathrm{~cm}$ long, it lies in the ground plane and begins at the projection of the original position of the vision system's origin onto that plane. Figure (b) is a view of the $3 \mathrm{D}$ line data, recovered from full stereo processing, from above.

Figure (c) shows the motion of the vehicle according to its own internal model as it attempts to navigate the path using the PID controller. This path is also show in (d) viewed from above and enlarged. Additionally the path of the vehicle according to the visual feedback (obtained at approximately $0.05 \mathrm{~Hz}$ ) is also shown (projected into the ground plane as visual feedback actually gives data with six degrees of freedom). This path, whilst not smooth, shows that stereo based geometrical information is (for this test object at least) able to provide positional information that is of good quality, and additionally, the extent to which the vehicle's path is in error.

Figures (e) and (f) illustrate the inclusion of visual information into the vehicle control loop. Notice that the vehicle controller's internal model now includes a number of discontinuities at points where visual data is incorporated (of course the actual motion of the vehicle is continuous). The visual feed back is sufficient to enable the vehicle to maintain a course that does not deviate from the required path.
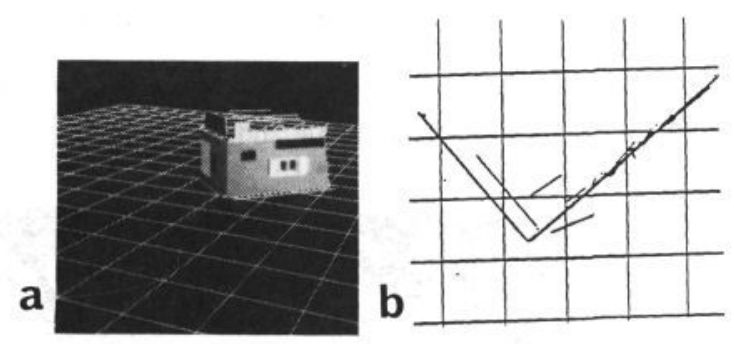

C
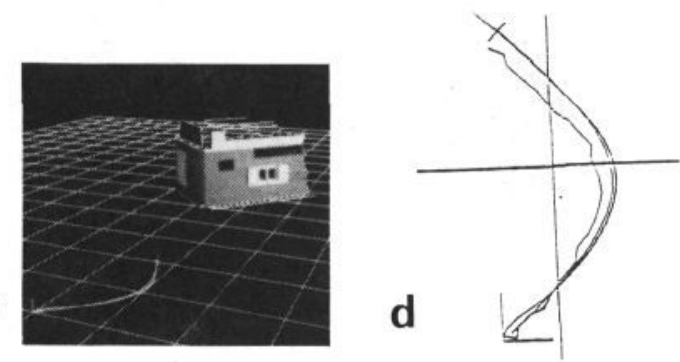

e
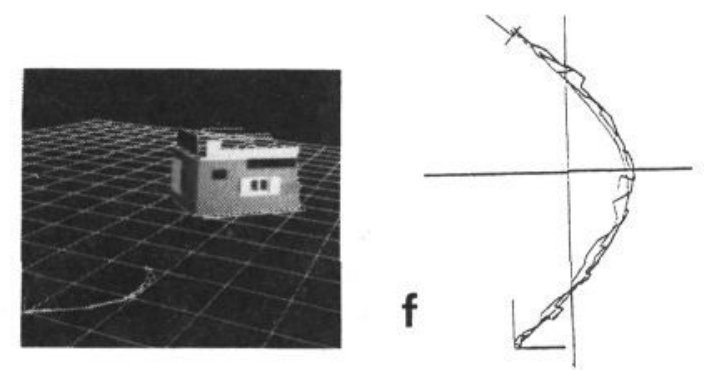

Future Developments.

Future developments are many fold. Problems which are being addressed currently include.

(1) The changing description of the world as a vehicle passes through it has many consequences. As a result of lighting, attitude and image blur the descriptions of primitives will slowly evolve; and this should be modeled.

(2) Errors in the calibration of the stereo system will be noticeable over long range motions, to the effect that as an object is approached it will appear to distort. This may also result in apparently safe paths becoming dangerous. Accordingly it may be necessary to account for and correct calibration errors.

(3) Tracking is only possible using the simple mechanisms above if (a) the errors from stereo are small in comparison to the time steps, (b) within the tolerance of the system, and (c) the system is robust. These conditions cannot be guaranteed to hold. Accordingly it will be necessary to incorporate more sophisticated tracking and position update mechanisms based upon a Kalman filter.

A transputer based multiprocessor system is currently under development. The system will utilise dual-ported video memory to distribute multiple regions of interest into the processing array at frame rate. It is expected that full scene descriptions will be recovered in a small number of seconds with focussed feature tracking at near frame rate.

\section{REFERENCES}

1. Porrill J., Pollard S.B. and Mayhew J.E.W. (1987), Optimal combination of multiple sensors including stereo vision, Image and Vision Computing, Vol 5, No 2, 175180.

2. Faugeras O.D., Ayache N. and Faverjon B. (1986). Building visual maps by combining noisy stereo measurements. Proc. Int. Conf. on Robotics and Automation, San Francisco, pp. 1433-1438.

3. Brooks R.A. (1986), A layered intelligent control system for a mobile robot, T.ird Int. Symp. on Robotics Res., 365372., MIT Press, Eç.s. Faugeras O and Giralt G.

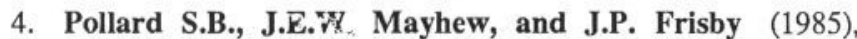
PMF: a stereo correspondence algorithm using a disparity gradient limit, Perception, 14, 449-470.

5. Canny J. (1986), A computational approach to edge detection, Trans. Patt. Anal. \& Mach. Intell, 679-698, PAMI-8.

6. Pearson K. (1901), On Lines and Planes of Closest Fit to Systems of Points in Space, Phil. Mag. VI, 2, pp 559.

7. Ballard D. H. and C. M. Brown (1982) Computer Vision, Prentice-Hall, New Jersey.

8. Ayache N, O.D. Faugeras, B. Faverjon and G. Toscani (1985) Matching depth maps obtained by passive stereo, Proc. Third Workshop on Computer Vision: Representation and Control, 197-204.

9. Porrill J. (1988), Optimal combination and constraints for geometrical sensor data, Int Journal on Robotics Res., special issue on sensor data fusion, Vol 7, 66-77.

Figure 7. 